\title{
Diferencias de mortalidad infantil dentro de Bolivia
}

\author{
Christian Darras ${ }^{1}$
}

RESUMEN El descenso de la mortalidad infantil registrado en Bolivia entre los censos nacionales de población de 1976 y 1992 encubre en realidad tendencias diferentes entre departamentos, así como entre zonas urbanas y rurales. Para poner de manifiesto esta situación, en 1996 se aplicó el análisis de las diferencias de mortalidad reducibles, que consiste en comparar la tasa de mortalidad infantil de cada departamento del país con la tasa mínima observada en los demás departamentos y calcular la reducción posible considerando comportamientos idénticos entre departamentos. Se procedió de manera similar para comparar las zonas urbanas con las rurales.

Los resultados muestran que en cuatro departamentos de Bolivia (Beni, Oruro, Pando y Potosí) hubo un aumento de las diferencias de mortalidad en relación con los demás departamentos. Es decir, que en esos departamentos la mortalidad infantil no se ha reducido como era de esperar teniendo en cuenta la evolución general de ese indicador en el país. Asimismo, las zonas rurales de todos los departamentos tienen diferenciales negativas en relación con las zonas urbanas. La cuantificación de esas diferencias permite proponer metas para la politica nacional de desarrollo humano sostenible.

En Bolivia, al igual que en los demás países de las Américas, durante las últimas décadas se ha observado un descenso sostenido de las tasas de mortalidad infantil. Sin embargo, al mismo tiempo ha aumentado la conciencia de que esa mejoría no ha sido igual para todos los habitantes y de que no se ha alcanzado una reducción paralela de las desigualdades en todas partes del país $(1,2)$.

Para describir más claramente esa evolución, la Organización Panamericana de la Salud ha elaborado instrumentos de medición que intentan reflejar la complejidad de la situación. Uno de ellos consiste en el análisis de

\footnotetext{
Ex experto asociado a la Oficina del Representante de la OPS en La Paz, Bolivia. Dirección postal: Casilla 11587,Calle \# 4, núm. 2 - Auquisamaña, La Paz, Bolivia.
}

las diferencias de mortalidad reducibles, cuyo fin es mostrar "las diferenciales que pueden ser reducidas en relación con un patrón de comparación" (3). Ese análisis parte de la observación de que los resultados en cuanto a condiciones de salud varían mucho de un país a otro y de que esa variación no puede explicarse solamente por diferencias en el crecimiento económico alcanzado por cada uno de ellos. Países con un mismo grado de crecimiento pueden presentar diferencias, a veces importantes, respecto a los valores de la mortalidad infantil y otros indicadores de salud. En esos casos, el país con peores condiciones de salud suele mostrar una diferencia de mortalidad que podría ser reducida cambiando algunos patrones de organización en el campo social, como el del acceso de la población a los servicios de educación y salud. Es así como necesita ser medido el impacto que tienen las reformas del sector de la salud sobre los grupos de población más pobres y los de las zonas rurales (4).

De manera similar, en Bolivia se observan tasas de mortalidad infantil bastante disímiles entre sus nueve departamentos. Por otra parte, la evolución de esas tasas tampoco ha sido pareja. Existen, por lo tanto, diferencias de mortalidad reducibles entre esos departamentos. Esas diferencias son también evidentes entre las zonas urbanas y rurales, en desmedro de las últimas. El objetivo del presente trabajo fue cuantificar la importancia de tales diferencias, con el propósito de orientar la formulación de la política de salud del país y facilitar así el establecimiento de metas concretas para reducir la mortalidad infantil. 


\section{MATERIALES Y MÉTODOS}

\section{Análisis de las diferencias entre localidades}

Para fines del presente estudio, en 1996 se realizó una comparación entre los departamentos de Bolivia utilizando la tasa de mortalidad infantil como indicador de la situación de salud. Por un lado, esa tasa es un indicador generalmente aceptado con esa finalidad y, por el otro, en Bolivia se dispone de los datos correspondientes procedentes de los censos nacionales de población de 1976 y 1992, lo que permite una comparación en el tiempo.

Las diferencias se calcularon utilizando el método presentado en la publicación Las condiciones de salud en las Américas (3). Ese método consiste en restar a la tasa específica de mortalidad infantil de un departamento la tasa más baja de todo el país para el mismo año y dividir luego el resultado por la tasa específica del departamento. Se obtiene así la proporción de la tasa específica para ese departamento que podría ser reducida, o sea la "diferencia" $u$ horizonte posible de reducción de la mortalidad infantil.

Los mismos resultados de los censos de 1976 y 1992 permitieron comparar también las situaciones urbana y rural dentro de cada departamento. En este caso, la diferencia de mortalidad reducible se calculó restando la tasa de la zona urbana de la tasa correspondiente a la zona rural y dividiendo luego el resultado por la tasa de la zona rural, visto que en todos los casos las tasas de mortalidad infantil son mayores en las zonas rurales.

\section{Análisis de las muertes infantiles evitables}

En ambos casos (comparación entre departamentos y comparación urbanorural) puede estimarse el número de muertes infantiles evitables. Para tal efecto, se procedió a multiplicar el número de defunciones de menores de un año de edad por el valor de la diferencia de mortalidad reducible del mismo año (en proporción). En efecto, en el caso de las diferencias entre departamentos, esa proporción representa precisamente el progreso en reducción de la mortalidad que se obtendría si el departamento considerado se comportase como el mejor del país en su conjunto. En el caso de las diferencias urbanorrurales, el progreso se mide con respecto a las muertes evitables como si los comportamientos fuesen iguales entre las zonas urbanas y rurales, dentro de cada departamento.

\section{RESULTADOS}

\section{Diferencias entre departamentos}

En el cuadro 1 se presentan las tasas de mortalidad infantil (TMI) respectivas de cada departamento estimadas para los años 1976 y 1992, con base en los censos nacionales de población correspondientes (5). Se presentan también las diferencias de mortalidad reducibles.

Se observa que, a pesar de una reducción global de las tasas de mortalidad infantil, el comportamiento en relación con las diferencias varió de un departamento a otro. En algunos departamentos aumentaron sus diferencias, como es el caso de Beni, Pando, Oruro y Potosí (figura 1). Beni presentó el aumento mayor y dejó de ser el departamento con la tasa de mortalidad infantil más baja de todo el país como lo era en 1976. Lo sustituyó el departamento de Santa Cruz.

Los otros departamentos (Santa Cruz, La Paz, Tarija, Cochabamba y Chuquisaca) experimentaron un descenso en sus diferencias, siendo los departamentos de Cochabamba y Tarija los que presentaron el mayor descenso.

De manera general, los incrementos de diferencias fueron más importantes que las reducciones. El promedio de incremento fue de 0,22 punto, mientras que el de descenso fue de 0,06 punto. Como resultado de esta evolución, en 1992 Potosí presentó la mayor diferencia $(0,52)$, seguido de cerca por Oruro $(0,50)$; por el contrario, la menor diferencia correspondió a Tarija $(0,05)$, considerando a Santa Cruz como valor de referencia nacional.

En el mismo cuadro se muestra el resultado del cálculo de muertes infantiles evitables, tomando las cifras de defunciones de menores de un año que arrojó el censo de 1992. El total de muertes infantiles evitables sería igual a 9888 . Esta cifra representa $25,1 \%$ de los fallecimientos correspondientes a aquel año. En términos absolutos, el número más importante de muertes evitables se observó en La Paz, Cochabamba y Potosí. Juntos, suman 7017 de ellas, o sea $71 \%$ del total.

CUADRO 1. Diferencias de mortalidad infantil reducibles y muertes infantiles evitables, por departamento. Bolivia, 1976-1992

\begin{tabular}{lrrrrrr}
\hline & \multicolumn{2}{c}{$\begin{array}{c}\text { TMla } \\
\text { (por 1 000) }\end{array}$} & \multicolumn{2}{c}{ Diferencia } & Defunciones & Muertes \\
\cline { 2 - 7 } Departamento & 1976 & 1992 & 1976 & 1992 & $<1$ año (1992) & evitables (1992) \\
\hline Beni & 114 & 90 & 0 & 0,37 & 1064 & 394 \\
Chuquisaca & 190 & 88 & 0,40 & 0,35 & 3047 & 1066 \\
Cochabamba & 174 & 78 & 0,34 & 0,27 & 5979 & 1614 \\
La Paz & 146 & 70 & 0,22 & 0,19 & 8623 & 1638 \\
Oruro & 162 & 113 & 0,30 & 0,50 & 2634 & 1317 \\
Pando & 136 & 85 & 0,16 & 0,33 & 140 & 46 \\
Potosí & 191 & 118 & 0,40 & 0,52 & 7241 & 3765 \\
Santa Cruz & 120 & 57 & 0,05 & 0 & 9765 & 48 \\
Tarija & 129 & 60 & 0,12 & 0,05 & 952 & 48 \\
\hline
\end{tabular}

a $\mathrm{TMl}=$ Tasa de mortalidad infantil.

Fuente: Bolivia, Instituto Nacional de Estadística. 
FIGURA 1. Mapa de Bolivia que muestra la ubicación de los distintos departamentos

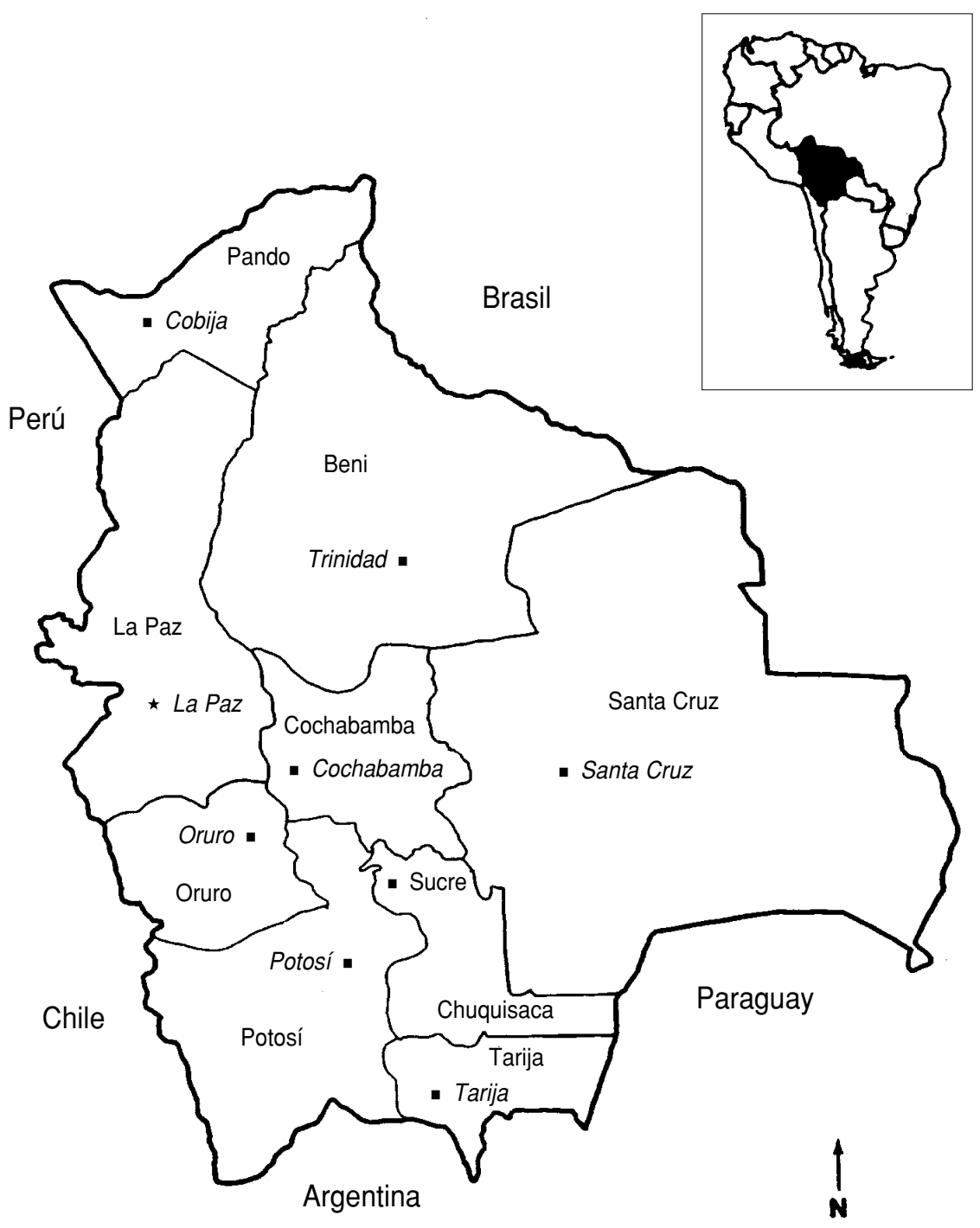

\section{Diferencias urbanorrurales}

El cuadro 2 muestra las tasas de mortalidad infantil en las zonas urbanas y rurales, por departamento, así como las respectivas diferencias de mortalidad reducibles. Presenta también el número de muertes evitables, basándose en defunciones de menores de un año en las zonas rurales, según datos del censo de 1992.

Puede observarse que solo en un departamento (Cochabamba) se produjo una reducción de la diferencia urbanorural. En cuatro otros departamentos (La Paz, Pando, Oruro y Potosí), la situación era estable. En los cuatro de- partamentos restantes (Santa Cruz, Beni, Tarija y Chuquisaca), las diferencias urbanorrurales se fueron acentuando, con un mayor incremento en Santa Cruz. Estos comportamientos diversos hacen que, en 1992, los departamentos con una mayor diferencia urbanorrural fueran Chuquisaca $(0,48)$, Santa Cruz $(0,41)$ y Cochabamba $(0,40)$, mientras que la menor diferencia se observó en Oruro $(0,16)$.

El total de muertes infantiles evitables sería, en el caso de estas diferencias, de 6665 , o sea $16,9 \%$ del total de defunciones de aquel año. En términos absolutos, las cifras más elevadas fueron las de Cochabamba, Potosí, Chu- quisaca y Santa Cruz, con un total de 5196 muertes evitables (78\% del total).

\section{DISCUSIÓN}

El análisis de las diferencias de mortalidad infantil reducibles en Bolivia permite afirmar que las diferencias más importantes correspondieron a los departamentos de Beni, Pando, Oruro, Potosí y Chuquisaca. Con la excepción de este último, fue también en esos departamentos donde las diferencias aumentaron más entre 1976 y 1992. Oruro y Potosí son departamentos esencialmente mineros, y el período considerado coincide con el derrumbe de esa actividad económica y el consecuente despido de millares de trabajadores. Por otro lado, Beni y Pando corresponden a la parte amazónica del país. Su población es la más dispersa y la organización de los servicios públicos es poco eficiente. Además, hay que mencionar que la economía local depende de la exportación (ganadería y explotación forestal) y que la presencia de pueblos indígenas representa un grupo de población marginada. Contrasta la situación de estos dos departamentos con la de Santa Cruz, también situado en el oriente de Bolivia pero que ha experimentado un importante crecimiento demográfico y económico en las últimas décadas. Algo similar sucede en Tarija, cuya diferencia de mortalidad es muy reducida en la actualidad. Sin embargo, la situación de La Paz, Cochabamba y Chuquisaca va mejorando con el tiempo, aunque con diferencias de mortalidad todavía importantes.

Los resultados aquí presentados se relacionan con la división observada en Bolivia entre departamentos ricos y departamentos pobres. Los primeros corresponden al llamado "eje central" (La Paz, Cochabamba y Santa Cruz), donde se concentran los mayores asentamientos de población y la mayor actividad económica (6). A este grupo se asocian Tarija y Chuquisaca, pero con una situación más precaria en este último departamento. Al margen quedan, por un lado, los departamentos mineros de 
CUADRO 2. Diferencias de mortalidad infantil reducibles entre zonas urbanas y rurales y muertes infantiles evitables. Bolivia, 1976-1992

\begin{tabular}{|c|c|c|c|c|c|c|}
\hline & & \multicolumn{2}{|c|}{$\begin{array}{c}\text { TMI } \\
\text { (por } 1000)\end{array}$} & \multicolumn{2}{|c|}{$\begin{array}{l}\text { Diferencia } \\
\text { urbanorrural }\end{array}$} & $\begin{array}{c}\text { Muertes } \\
\text { evitables }\end{array}$ \\
\hline \multicolumn{2}{|c|}{ Departamento } & 1976 & 1992 & 1976 & 1992 & 1992 \\
\hline \multirow[t]{2}{*}{ Beni } & Zona urbana & 99 & 77 & & & \\
\hline & Zona rural & 122 & 109 & 0,19 & 0,29 & 132 \\
\hline \multirow[t]{2}{*}{ Chuquisaca } & Zona urbana & 128 & 52 & & & \\
\hline & Zona rural & 201 & 100 & 0,36 & 0,48 & 1254 \\
\hline \multirow[t]{2}{*}{ Cochabamba } & Zona urbana & 115 & 56 & & & \\
\hline & Zona rural & 234 & 94 & 0,51 & 0,40 & 1664 \\
\hline \multirow[t]{2}{*}{ La Paz } & Zona urbana & 129 & 63 & & & \\
\hline & Zona rural & 161 & 81 & 0,20 & 0,22 & 840 \\
\hline \multirow[t]{2}{*}{ Oruro } & Zona urbana & 148 & 103 & & & \\
\hline & Zona rural & 176 & 123 & 0,16 & 0,16 & 254 \\
\hline \multirow[t]{2}{*}{ Pando } & Zona urbana & 94 & 60 & & & \\
\hline & Zona rural & 140 & 88 & 0,33 & 0,32 & 41 \\
\hline \multirow[t]{2}{*}{ Potosí } & Zona urbana & 158 & 98 & & & \\
\hline & Zona rural & 205 & 129 & 0,23 & 0,24 & 1259 \\
\hline \multirow[t]{2}{*}{ Santa Cruz } & Zona urbana & 102 & 46 & & & \\
\hline & Zona rural & 136 & 78 & 0,25 & 0,41 & 1019 \\
\hline \multirow[t]{2}{*}{ Tarija } & Zona urbana & 102 & 46 & & & \\
\hline & Zona rural & 144 & 74 & 0,29 & 0,38 & 202 \\
\hline
\end{tabular}

a TMl=Tasa de mortalidad infantil.

Fuente: Bolivia, Instituto Nacional de Estadística.

Oruro y Potosí y, por el otro, los amazónicos de Beni y Pando. La diferencia entre los dos grupos se acentuó durante el período de 1976 a 1992.

Una igualación de los comportamientos entre los departamentos permitiría reducir la mortalidad infantil en $25 \%$, suponiendo un comportamiento de todos los departamentos del país comparable al de Santa Cruz. En cifras absolutas, las muertes evitables más importantes se observarían en La Paz, Cochabamba y Potosí. Por lo tanto, desde el punto de vista de la cantidad total de muertes evitables, esos departamentos serían prioritarios aun cuando la situación estuviera evolucionando más favorablemente en La Paz y Cochabamba que en Potosí.

El estudio de las diferencias urbanorurales revela otra dimensión de las desigualdades nacionales. Estas diferencias son similares a las que existen entre departamentos $(17 \%$ frente a $25 \%$ ). La igualación del comportamiento entre las zonas rurales y urbanas tendría un impacto sobre la morta- lidad infantil similar al de la igualación entre departamentos.

Para un niño, el riesgo de morir se relaciona tanto con su condición rural como con su residencia en un departamento dado. Además, cabe notar que la situación de la mortalidad infantil se estancó, cuando no empeoró, durante el período de 1976 a 1992, con la única excepción de Cochabamba. La desigualdad de condiciones entre zonas urbanas y rurales es entonces un hecho trascendental para la definición de una política de salud. Asimismo, estas cifras ponen de manifiesto que los resultados generales observados a nivel de los departamentos deben ser interpretados en función del grado de urbanización de la población. Por ejemplo, a pesar de que Tarija tiene tasas de mortalidad infantil iguales o inferiores a las de Santa Cruz, cuando se desagregan por urbana y rural, la tasa general global es superior, lo que refleja la presencia de una población más predominantemente urbana en el segundo departamento que en el primero.
Este análisis de las diferencias de mortalidad infantil no ha descubierto una situación desconocida anteriormente en Bolivia. La existencia de desigualdades marcadas entre departamentos, así como entre zonas urbanas y zonas rurales, es una realidad palpable para quien revise las cifras existentes o recorra el país. Sin embargo, el análisis permite cuantificar las diferencias y proponer metas realistas. La cifra de reducción de la mortalidad no se basa en estimaciones arbitrarias, sino en resultados concretos ya obtenidos, en el mismo país, en condiciones similares.

El presente tipo de análisis depende obviamente de la fiabilidad de los datos censales. Aun sabiendo que existen márgenes de error en ellos y que además esos márgenes no son constantes, lo importante no es pretender llegar a conclusiones estadísticamente significativas sino a observar tendencias generales. En este sentido, el análisis permite también identificar cambios en el tiempo, gracias a la comparación de la situación en épocas diferentes. En este caso, contribuye a definir una articulación de las políticas económica y social para el desarrollo armonioso del país y, en especial, la relación entre el crecimiento económico y los beneficios sociales (7). Para realizar estudios de diferencias más relacionados con los modelos mismos de organización de los servicios de salud, se podrían utilizar indicadores con mayor especificidad de la eficacia de esos servicios, como la mortalidad neonatal o la mortalidad materna.

Agradecimiento. El autor expresa su reconocimiento a Zulema Lozano por su apoyo en la reunión de los datos básicos y a Graciela Uriburu (Oficina de la Representación de la OPS en Bolivia) y Marlo Libel, Norberto Dachs y Patricia Ruiz (Programa de Análisis de la Situación de Salud, OPS) por sus valiosos comentarios. 


\section{REFERENCIAS}

1. Roses M. Condiciones de salud y vida en Bolivia. En: Darras C, Meinardus M, eds. Pobreza y salud en Bolivia. La Paz: OPS/OMS, Misión de Cooperación Técnica Holandesa (MCTH) e Instituto Latinoamericano de Investigaciones Sociales (ILDIS); 1994

2. Wolowyna O, Pinto G. Sobrevivencia infantil en Bolivia. La Paz: Consejo Nacional de Población (CONAPO); 1990.

3. Organización Panamericana de la Salud. Las condiciones de salud en las Américas: edición de 1994. Washington, DC: OPS; 1994. (Publicación científica 549).
4. Mesa-Lago C. Atención de salud para los pobres en la América Latina y el Caribe. Washington, DC: Organización Panamericana de la Salud y Fundación Interamericana; 1992. (Publicación científica 539).

5. Bolivia, Ministerio de Planeamiento y Coordinación, Instituto Nacional de Estadística. Censo nacional de población y vivienda 1992 resultados finales. La Paz; 1993.

6. Institute for European-Latin American Relations. Bolivia: the difficult process of transformation. Dossier No. 57. Madrid, septiembre de 1996.
7. Comisión Económica para América Latina y el Caribe (CEPAL) / Centro Latinoamericano de Administración para el Desarrollo (CLAD)/ Sistema Económico Latinoamericano (SELA). Desarrollo con equidad: hacia una nueva articulación de políticas económicas y sociales en América Latina y el Caribe. Caracas. Editorial Nueva Sociedad; 1996.

Manuscrito recibido el 7 de marzo de 1997 y aceptado en versión revisada el 18 de marzo de 1998.

ABSTRACT There was an overall decrease in infant mortality in Bolivia between the national censuses of 1976 and of 1992. That general pattern, however, in fact conceals differences in trends from one department to another, as well as between urban and rural areas. In order to elucidate this situation, in 1996 the reducible differences in mortality were analyzed. This was done by comparing the infant mortality rate in each department of the country with the lowest rate found in the other departments and calculating the possible reduction if all the departments had had the same level of achievement. Using a similar approach, urban areas were compared with rural ones.

The results show that in four departments of Bolivia (Beni, Oruro, Pando, and Potosí) the differences in mortality rates increased in comparison to the other departments. That is, in those four departments infant mortality has not fallen as much as could be expected given the general trends in the country. Similarly, the decline in infant mortality rates in the rural areas has lagged behind the decreases seen in urban areas. Quantifying these differences makes it possible to propose goals for the national policy of sustainable human development. 